\title{
Synthesis, structure, theoretical studies and luminescent properties of a ternary erbium(III) complex with acetylacetone and bathophenanthroline ligands
}

Pablo Martín-Ramos ${ }^{1,2}$, Pedro S. Pereira Silva ${ }^{1} *$, Pedro Chamorro-Posada ${ }^{3}$, Manuela Ramos Silva ${ }^{1}$, Bruce F. Milne ${ }^{4,5}$, Fernando Nogueira ${ }^{4}$ and Jesús Martín-Gil ${ }^{2}$

CEMDRX, Department of Physics, Universidade de Coimbra, Rua Larga, P-3004-516 Coimbra, Portugal.*E-mail: psidonio@pollux.fis.uc.pt.

2 Advanced Materials Laboratory, ETSIIAA, Universidad de Valladolid, Avenida de Madrid 44, 34004 Palencia, Spain

3 Higher Technical School of Telecommunications Engineering, Universidad de Valladolid, Campus Miguel Delibes, Paseo Belén 15, 47011 Valladolid, Spain.

4 Centre for Computational Physics, Department of Physics, Universidade de Coimbra, P-3004516 Coimbra, Portugal.

5 Donostia International Physics Centre, Paseo Manuel de Lardizabal 4, 20018 Donostia-San Sebastián, Spain.

\begin{abstract}
A novel erbium(III) complex with acetylacetone (Hacac) and bathophenanthroline (4,7diphenyl-1,10-phenanthroline, bath) ligands, formulated as [ $\left.\operatorname{Er}(\mathrm{acac})_{3}(\mathrm{bath})\right]$, has been characterized by elemental analysis, X-ray diffraction, thermogravimetric analysis, Fourier transform infrared spectroscopy, Raman spectroscopy, absorption and emission spectroscopies. In the theoretical part of this study, semi-empirical quantum chemistry methods using AM1, PM3, PM6 and PM7 models have been employed to predict the structure of the complex, calculate the geometric and crystallographic parameters, and make comparisons with spectroscopic data using INDO/S-CI calculations. Real-time time-dependent density-functional theory (TDDFT) has also been used to calculate the optical absorption spectrum of the complex in the gas phase.
\end{abstract}

Keywords: $\beta$-diketonate; erbium(III); MOPAC; Sparkle; TDDFT.

\section{INTRODUCTION}

Different approaches for the design of lanthanide coordination compounds as potential precursors for electroluminescent device thin films have been proposed [1]. In fact, formation of mixed ligand complexes is a well-known methodology so as to modify the different properties of lanthanide compounds. The composition and the structure of such compounds strongly depend on the electronic features of the ligands. In this respect, it is very important to analyse the changes in structural parameters, bond energies and other properties of lanthanide complexes as a function of ligand types. A solution to these tasks lies in combining experimental and theoretical characterization resources. In theoretical chemistry, the ability to predict geometries of lanthanide complexes is fundamental in the design of luminescent complexes, for which theoretical models such as Sparkle [2], the combined quantum mechanical/molecular mechanical (QM/MM) technique $[1,3]$ and the universal force field for the MM part have been successfully used so as to model the structures and properties of $\beta$-diketonate-based lanthanide complexes. As a counterpart to this 
theoretical approach, our research team has set the goal of conducting an experimental study on the structure and properties of erbium $\beta$-diketonate-based mixed ligand complexes with $\mathrm{N}, \mathrm{N}$-donor molecules [4-11].

The choice of $\beta$-diketonates amongst the various strategies aimed at improving lanthanide luminescent quantum efficiencies discussed in the literature [12] is supported by their wide commercial availability and by their remarkable synthetic versatility. In this study the simplest $\beta$ diketonate (acetylacetonate, acac), where the substituents on both carbonyl groups are methyl groups, has been chosen to exemplify the numerical techniques. Nevertheless, the substitution of these $\mathrm{CH}_{3}$ groups by other groups would allow to control and enhance the properties of the corresponding rare-earth complexes (for instance, branched alkyl chains are known to increase the solubility in organic solvents and the volatility, perfluorinated alkyl groups increase the Lewis acidity, aromatic substituents increase the light absorption in comparison to aliphatic substituents, etc.). Such flexibility has allowed the successful application of analogous erbium(III) complexes as, for example, active layers in organic light-emitting diodes [13], as dopants in liquid-crystals aimed at LCDs [14] or in other matrices with a view to optical amplification [15-20].

With regard to the role of the N,N-donor ligands, they act as Lewis bases and complete the rare-earth ion's first coordination sphere. Two very popular diimides are 1,10-phenanthroline (phen) and 2,2'-bipyridine (bipy). In the novel complex reported herein, bathophenanthroline (bath) has been chosen instead. Being more basic than phen $(\mathrm{pKa}=4.27)$ or bipy $(\mathrm{pKa}=4.38)$, bath $(\mathrm{pKa}=4.67)$ would be expected to contribute more electron density through $\mathrm{Er}-\mathrm{N}$ bond making the $\mathrm{Er}^{3+}$ ion more electron rich. As a result, the $\mathrm{Er}^{3+}$ will show less attraction for electron donor solvents. The rigidly planar structure of bath would also be helpful in obstructing the coordination of solvent molecules to inner coordination sphere of $\mathrm{Er}^{3+}$ and restricting the complex-solvent interaction [21]. This should help to decrease non-radiative losses. Secondly, the bulkier and more rigid planar structure of bathophenanthroline should cause a higher intensity of the sensitized luminescence, because such structure allows a better energy transfer [21-24].

In this paper, the erbium(III) complex formulated as $\left[\operatorname{Er}(\mathrm{acac})_{3}(\mathrm{bath})\right]$; has been thoroughly characterized by elemental analysis, X-ray diffraction, thermogravimetric analysis, Fourier transform infrared spectroscopy, Raman spectroscopy, absorption spectroscopy and luminescence in solid state. Part of this data has then been used as a reference in order to evaluate the suitability of several semi-empirical calculation methods for predicting the equilibrium energy configuration and -in combination with INDO/S method and CIS- the electronic properties of the complex. Finally, a comparison with more computationally-intensive TDDFT calculations for the optical absorption spectrum has been conducted.

\section{EXPERIMENTAL AND COMPUTATIONAL METHODS}

\subsection{Materials, synthesis and analytical data}

All reagents and solvents employed were commercially available and used as supplied without further purification. All the procedures for complex preparation were carried out under nitrogen environment and using dry reagents to avoid the presence of water and oxygen, which can quench metal photoluminescence (PL).

Tris(acetylacetonate)mono(bathophenanthroline)erbium(III), [Er(acac) $)_{3}($ bath)], was obtained by mixing of equimolar quantities of methanol solutions of erbium(III) 2,4-pentanedionate [erbium(III) acetylacetonate hydrate 97\%, CAS No. 70949-24-5, Sigma Aldrich] (0.464 g, 1 mmol) and bathophenanthroline [4,7-diphenyl-1,10-phenanthroline 97\%, CAS No. 1662-01-7, Sigma Aldrich] $(0.332 \mathrm{~g}, 1 \mathrm{mmol})$. The mixture was heated to $75^{\circ} \mathrm{C}$ and stirred overnight, then washed with dioxane, and finally dried in vacuum to give the product in $86.2 \%$ yield (based in Er). Crystals 
suitable for X-ray analysis were obtained by slow evaporation of a methanol-dioxane solution at room temperature (RT).

Chemical formula: $\mathrm{C}_{39} \mathrm{H}_{37} \mathrm{ErN}_{2} \mathrm{O}_{6} . \mathrm{M}_{\mathrm{W}}$ : 796.98. Anal. Calcd. For $\mathrm{C}_{39} \mathrm{H}_{37} \mathrm{ErN}_{2} \mathrm{O}_{6}: \mathrm{C}, 58.77 ; \mathrm{H}$, 4.68; Er, 20.99; N, 3.51; O, 12.05. Found: C, 58.81; H, 4.66; N, 3.45\%.

\subsection{Physical and spectroscopic measurements}

C, H, N elemental analyses were conducted using a Perkin Elmer CHN 2400 apparatus.

Differential scanning calorimetry (DSC) data were obtained on a DSC TA instrument mod Q100 v.9.0 with a heating rate of $10^{\circ} \mathrm{C} / \mathrm{min}$ under a $\mathrm{N}_{2}$ atmosphere. Thermogravimetric and differential thermal analyses were carried out in an inert atmosphere with a Perkin Elmer STA 6000 Simultaneous Thermal Analyzer, by heating $7 \mathrm{mg}$ of sample in a slow stream of $\mathrm{N}_{2}(20 \mathrm{~mL} / \mathrm{min})$ from room temperature up to $800^{\circ} \mathrm{C}$, with a heating rate of $20^{\circ} \mathrm{C} / \mathrm{min}$.

Infrared spectrum was recorded with a Thermo Nicolet 380 FT-IR spectrometer in $\mathrm{KBr}$ pellets.

Raman spectrum was recorded with a FT-Raman Bruker FRA106 by using a near-IR (Nd: YAG, $1064.1 \mathrm{~nm}$ ) laser to excite the sample.

The crystal structure was elucidated by X-ray diffraction analysis. Prior to structural characterisation, the powder diffractogram of the erbium complex was obtained using an ENRAFNONIUS powder diffractometer (Deby-Scherrer geometry) equipped with an INEL CPS120 detector and a quartz monochromator selecting the $\mathrm{Cu} \mathrm{K} \alpha 1$ wavelength (transmission geometry, capillary of $0.3 \mathrm{~mm}$ diameter).

For the determination of crystal structure by X-ray diffraction, a crystal of [Er(acac $\left.)_{3}(\mathrm{bath})\right]$ was glued to a glass fibre and mounted on a Bruker APEX II diffractometer. Diffraction data was collected at room temperature 293(2) K using graphite monochromated $\operatorname{MoK} \alpha(\lambda=0.71073 \AA)$. Absorption corrections were made using SADABS [25]. The structure was solved by direct methods using SHELXS-97 [26] and refined anisotropically (non-H atoms) by full-matrix leastsquares on $F^{2}$ using the SHELXL-97 program [26]. PLATON [27] was used to analyse the structure. Mercury version 3.1 [28] was used for figure plotting. Atomic coordinates, thermal parameters and bond lengths and angles have been deposited at the Cambridge Crystallographic Data Centre (CCDC). Any request to the CCDC for this material should quote the full literature citation and the reference number CCDC 966774.

The optical absorption spectrum of the product in the UV-Vis region was recorded with a Hitachi U-2010 spectrophotometer in methanol diluted solutions $\left(10^{-5}\right.$ and $\left.10^{-3} \mathrm{M}\right)$. The absorbance in the NIR range was recorded using a Varian 660-IR FT-IR spectrometer in $10 \mathrm{wt} \% \mathrm{KBr}$ pellet.

Steady state luminescence and excitation spectra for the samples in powder form were collected with a Jobin Yvon Fluorolog 3 spectrofluorometer equipped with a liquid nitrogen cooled InGaAs detector. The PL spectrum was recorded upon excitation at $350 \mathrm{~nm}$ and at ${ }^{2} \mathrm{H}_{11 / 2}\left(\lambda_{\text {exc }}=532\right.$ $\mathrm{nm}) \mathrm{Er}^{3+}$ absorption with a 450W Xenon lamp and a $500 \mathrm{~mW}$ continuous laser, respectively. The spectrum was acquired in the 850-1600 nm range with $1 \mathrm{~s}$ integration time at $1 \mathrm{~nm}$ steps. The NIR luminescence decay curve was measured upon excitation of the $\mathrm{Er}^{3+}$ ions at $980 \mathrm{~nm}$ with a Spectra Physics Quanta-Ray MOPO-730 laser system. The emitted light was dispersed using a singlegrating $0.34 \mathrm{~m}$ focal length spectrometer (Spex 340E) and detected with a Peltier-cooled, NIRextended photomultiplier (Hamamatsu H9170-75) and a lock-in amplifier. The lifetime was measured by using a digital oscilloscope (Tektronix TDS520). The optical absorption and photoluminescence spectra have been measured at room temperature and have been corrected by the spectral response of the experimental setups. 


\subsection{Computational methods}

\subsubsection{Semi-empirical calculations}

Using the experimental crystallographic data as an initial guess, the ground state geometries and the corresponding vibrational frequencies were obtained using the Sparkle/AM1 [29,30], Sparkle/PM3 [31], Sparkle/PM6 [32,33] and Sparkle/PM7 [34,35] models implemented in the MOPAC2012 software [36]. The computations were performed on a server with four AMD Opteron 16 Core processors and 128 GB of memory and a Linux operating system. No imaginary vibrational frequencies were found for any of the optimized geometries.

The electronic spectra for each of the optimized structures were calculated using the ORCA electronic structure package version 2.9.1 [37,38] via the intermediate neglect of differential overlap/spectroscopic (INDO/S) method and configuration interaction with singles (CIS) [39,40] replacing the $\mathrm{Er}^{3+}$ ion with a point charge as described by Andrade et al. [41]. Triplet excited states have also been calculated for the PM7 optimized geometry using the CIS module in ORCA software package.

Semi-empirical calculations were also performed in order to optimize the solid crystalline structures using a treatment of periodic boundary conditions [39] in the MOPAC2012 software [36]. In all the calculations, we used the keywords SPARKLE XYZ and GNORM=1: the Sparkle model to treat the $\mathrm{Er}^{3+}$ ions, Cartesian coordinates, and the same exit criteria (when the gradient norm drops below 1.0) reported by Oliveira et al. [42]. We replicated the crystallographic unit cell $l, m$ and $n$ times along the Cartesian axis $x, y$ and $z$, respectively. In each case, the keyword MERS $=(l, m, n)$ was used, where $l, m$ and $n$ could be either 1 or 2 in each calculation.

\subsubsection{TDDFT calculations}

We employed real-time time-dependent density-functional theory [43] (TDDFT) as implemented in the OCTOPUS code $[44,45]$ to calculate the optical absorption spectrum of $\left[\operatorname{Er}(\mathrm{acac})_{3}\right.$ (bath)] in the gas phase. In real-time TDDFT, the time-dependent Kohn-Sham equations are used to propagate the ground-state wavefunctions up to some finite time, thus allowing the determination of any observable of the system as a function of time. The optical absorption crosssection $\sigma(\omega)$ is related to the imaginary part of the dynamical polarizability $\alpha(\omega)$ :

$$
\sigma(\omega)=\frac{4 \pi \omega}{c} \mathfrak{J} \mathfrak{m}\{\langle\alpha(\omega)\rangle\}
$$

where the brackets denote orientational averaging. In order to obtain the optical absorption spectra, a dipolar perturbation:

$$
\delta v(\boldsymbol{r}, t)=-E_{j} x_{j} \delta(t)
$$

that acts at $t=0$ and equally excites all the frequencies of the system was applied, and we kept track of the dipole moment of the system as a function of time:

$$
\boldsymbol{p}(t)=\int d \boldsymbol{r} \boldsymbol{r} n(\boldsymbol{r}, t)
$$

where $n(\boldsymbol{r}, t)$ is the time-dependent electronic density. The components of the polarizability tensor are then simply obtained by Fourier transforming the induced dipole:

$$
\alpha_{i j}(\omega)=\frac{\delta p_{i}(\omega)}{E_{j}}
$$

The ground state of $\left[\mathrm{Er}(\mathrm{acac})_{3}(\mathrm{bath})\right]$ was obtained using standard density-functional theory, also as implemented in the code OCTOPUS. This code solves the (time-independent) Kohn-Sham equations on a real space grid, using pseudopotentials to get rid of the core electrons of the atoms. 
The Atomic Pseudopotentials Engine (APE) code [46] was used in order to obtain norm-conserving pseudopotentials of the Troullier-Martins type [47] for all the elements in the calculation.

In OCTOPUS, all the relevant functions are discretized in a real-space regular rectangular grid, and we chose the simulation box to be composed of spheres around each atom. Therefore, there are essentially two parameters that control the convergence of the spectra: the grid spacing and the radius of the spheres.

A uniform spacing of 0.20 atomic units and a sphere radius of 20 atomic units for $\mathrm{Er}^{3+}$ were used (the other elements' sphere radii were set at the default values of OCTOPUS for each element). The self-consistent electronic cycle was considered to have reached convergence when all the eigenvalues of the occupied states calculated in two consecutive iterations differed by less than $5.10^{-7}$ atomic units. These parameters also meant that the absorption spectra were converged to better than $0.1 \mathrm{eV}$. As for the choice of exchange and correlation functional we opted for the Perdew-Zunger [48] parameterization of the Local Spin Density approximation for both the ground state calculation and the time propagation. LSD is not the best choice for the optimization of the ground state geometry, but the structure was not re-optimized: all the calculations were done using the XRD unrelaxed geometry. For the calculation of the linear response, TDDFT requires only the exchange and correlation potential and not the energy, and this quantity is practically the same along the most popular approximations for exchange and correlation. This justifies the use of LSD also for the time propagation.

The spectra were obtained propagating the time-dependent Kohn-Sham equations for 180 atomic units of time, in time steps of 0.01 atomic units. As this propagation was not long enough to get sharp peaks from the Fourier transform of the dipole moment, we also used compressed sensing [49] in the analysis of the propagation.

\section{RESULTS AND DISCUSSION}

\subsection{Structural description}

$\left[\mathrm{Er}(\mathrm{acac})_{3}\right.$ (bath) $]$ crystallizes in the triclinic space group $P-1$, with cell parameters $a=11.8685(2) \quad \AA, \quad b=12.1053(3) \quad \AA, \quad c=14.2355(3) \AA \AA \quad \alpha=96.6630(10)^{\circ}, \quad \beta=105.0080(10)^{\circ}$, $\gamma=110.1770(10)^{\circ}, V=1806.05(7) \AA^{3}$ (Figure 1, Table 1). The lanthanide ion is eight-coordinated by six $\mathrm{O}$ atoms from the acetylacetonate anions and two $\mathrm{N}$ atoms from the bathophenanthroline molecule, forming a distorted square-antiprismatic structure in which the set N1, N2, O3, O4 and the set $\mathrm{O} 1, \mathrm{O} 2, \mathrm{O} 5, \mathrm{O} 6$ form two approximate squares, respectively (Figure 1). The trivalent erbium ion lies approximately in the middle of the antiprism with a distance of $1.39 \AA$ to the face containing the $\mathrm{N}$ atoms and $1.19 \AA$ to the opposite face containing exclusively oxygens. The angle between the least-squares planes of the square faces is just $2^{\circ}$. The Er-N distances are 2.545(2) and 2.550(2) $\AA$ and the Er-O distances are in the range 2.289(2)-2.313(2) $\AA$. The N-Er-N bite angle is

| $63.6^{\circ}$. Selected geometric parameters are given in Table 2 . 


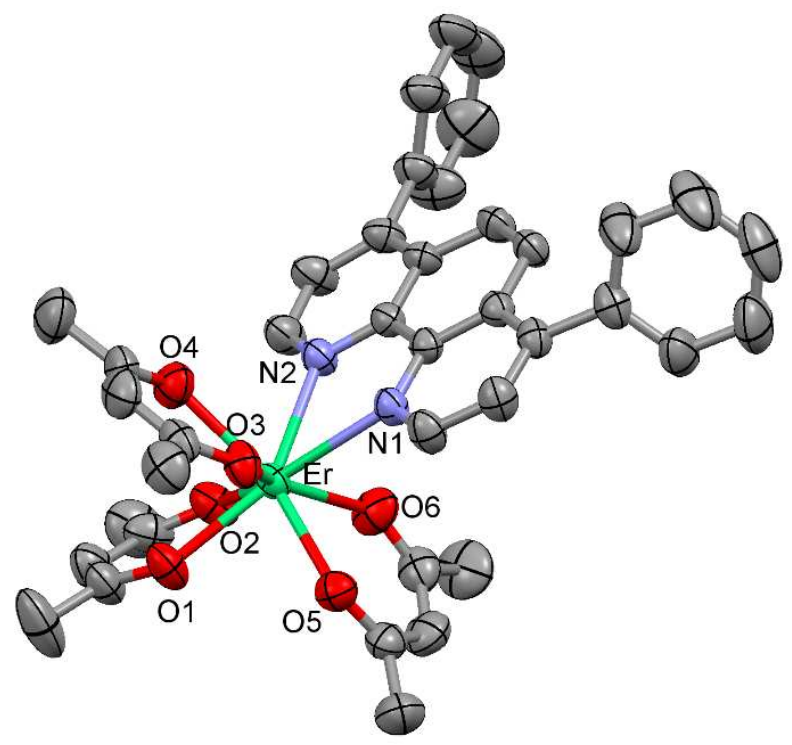

Figure 1. A perspective view of the eightfold coordination polyhedron of $\left[\operatorname{Er}(\mathrm{acac})_{3}(\mathrm{bath})\right]$, possessing a distorted square-antiprismatic geometry. Displacement ellipsoids are drawn at the $30 \%$ probability level. Hydrogen atoms have been omitted for clarity.

The six-membered chelate rings formed by $\mathrm{Er}^{3+}$ and the acetylacetonate ligands possess nearly planar geometries. The chelating ring associated with bathophenanthroline also possesses nearly planar geometry. The slight distortion of the $\beta$-diketonate rings occurs along the axis connecting the two oxygen atoms of the ligand. The bathophenanthroline molecules are not planar, as the terminal phenyl rings are rotated $61.36(15)$ and $70.29(17)^{\circ}$ with respect to the central phenanthroline unit.

The comparison of Er-O bond lengths in $\left[\mathrm{Er}(\mathrm{acac})_{3}(\mathrm{bath})\right]$ with the corresponding bonds lengths in $\left[\operatorname{Er}(\mathrm{acac})_{3}\right]$ is directly related to the interactions of this mono ligand complex with the additional ligand, bathophenathroline, whose introduction in the coordination sphere of erbium stimulates redistribution of electronic density in the ternary complex. The Er-bath bonding is not purely electrostatic and its formation is accompanied by a decrease in the Er-acac bond energy. Consequently, the Er-O bond lengths in $\left[\operatorname{Er}(\mathrm{acac})_{3}(\mathrm{bath})\right]$ are larger than that in $\left[\operatorname{Er}(\mathrm{acac})_{3}\left(\mathrm{H}_{2} \mathrm{O}\right)_{2}\right]$ $[50,51]$. The coordination geometry of $\left[\operatorname{Er}(\mathrm{acac})_{3}(\right.$ bath $\left.)\right]$ is very similar to that found in the related complex $\left[\operatorname{Er}(\mathrm{acac})_{3}(\mathrm{phen})\right][52]$. In $\left[\operatorname{Er}(\mathrm{acac})_{3}(\right.$ phen) $]$ the Er-N distances are 2.523(5) and 2.568(5) $\AA$, with a N-Er-N bite angle of $64.3^{\circ}$ and the Er-O distances range from $2.287(4)$ to $2.324(4) \AA$. 


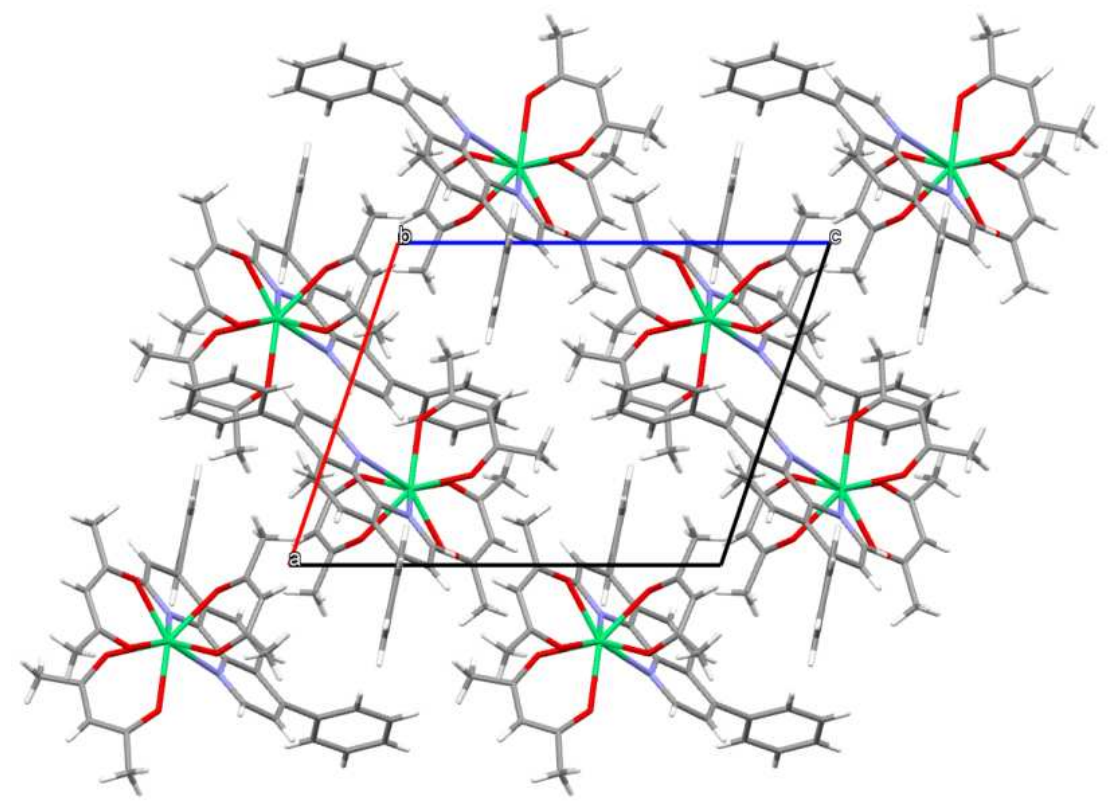

Figure 2. Packing diagram of $\left[\operatorname{Er}(\mathrm{acac})_{3}(\mathrm{bath})\right]$

Table 1. Crystal data and structure refinement

Complex

Empirical formula
Formula weight
Temperature
Wavelength
Crystal system
Space group

$a$
$b$
$c$
$\alpha$
$\beta$
$\gamma$
Volume
$Z$

Density (calculated)

Absorption coefficient $F(000)$

Crystal size

$\theta$ range for data collection Index ranges

Reflections collected

Independent reflections

Completeness to $2 \theta=55^{\circ}$

Refinement method

Data/restrains/parameters

Goodness-of-fit on $\mathrm{F}^{2}$

Final $R$ indices $[I>2 \sigma(I)]$

$R$ indices (all data)

Largest diff. peak and hole
[Er(acac $)_{3}$ (bath)]

$\mathrm{C}_{39} \mathrm{H}_{37} \mathrm{ErN}_{2} \mathrm{O}_{6}$
796.97
$293(3) \mathrm{K}$
$0.71073 \AA$
Triclinic
$P-1$
$11.8685(2) \AA$
$12.1053(3) \AA$
$14.2355(3) \AA$
$96.6630(10)^{\circ}$
$105.0080(10)^{\circ}$
$110.1770(10)^{\circ}$
$1806.05(7) \AA^{\circ}$
2
$1.466 \mathrm{~g} \mathrm{~cm}^{-3}$
$2.370 \mathrm{~mm}^{-1}$
802
$0.26 \times 0.18 \times 0.10 \mathrm{~mm}^{3}$
$1.93-30.64^{\circ}$
$-17<h<17 ;-17<k<17 ;-20<l<20$
46456
11070
$99.9 \%$
Full matrix LS on $\mathrm{F}^{2}$
$9229 / 0 / 439$
1.047
$R=0.0276 ; \mathrm{w} R=0.0651$
$R=0.0401 ; \mathrm{w} R=0.0724$
$-0.549 / 1.132$


Table 2. Selected distances and angles $\left(\AA^{\circ},{ }^{\circ}\right)$

\begin{tabular}{cccc} 
Bond & Distance & Bonds & Angle \\
\hline Er1-N1 & $2.550(2)$ & O1-Er1-O5 & $78.27(7)$ \\
Er1-N2 & $2.5454(19)$ & O1-Er1-O4 & $82.78(8)$ \\
Er1-O1 & $2.3038(18)$ & O5-Er1-O3 & $81.08(7)$ \\
Er1-O2 & $2.2897(19)$ & O4-Er1-O2 & $80.29(8)$ \\
Er1-O3 & $2.3114(18)$ & O3-Er1-O2 & $141.78(7)$ \\
Er1-O4 & $2.306(2)$ & O1-Er1-N1 & $146.72(6)$ \\
Er1-O5 & $2.2890(19)$ & O6-Er1-N1 & $77.17(8)$ \\
Er1-O6 & $2.313(2)$ & O6-Er1-N2 & $68.90(7)$ \\
Er1-N avg. & 2.548 & O5-Er1-N2 & $134.02(7)$ \\
Er1-O avg. & 2.302 & & \\
\hline
\end{tabular}

\subsection{Assessment of the accuracy of semi-empirical quantum chemistry methods}

The comparison between the results obtained with the four models studied reveal that a substantial improvement in accuracy is obtained with the most recent Sparkle/PM7 model [34,35]. With this model, the unsigned mean error (UME) in the calculations for a single unit cell is $\mathrm{UME}_{a, b, c}=0.89 \%$ for the $a, b$ and $c$ parameters and $\mathrm{UME}_{\alpha, \beta, \gamma}=0.39 \%$ for the $\alpha, \beta$ and $\gamma$ parameters. For Sparkle/AM1, $\mathrm{UME}_{a, b, c}=7.61 \%$ and $\mathrm{UME}_{\alpha, \beta, \gamma}=3.12 \%$; for Sparkle/PM3 $\mathrm{UME}_{a, b, c}=0.97 \%$ and $\mathrm{UME}_{\alpha, \beta, \gamma}=3.32 \%$; and for Sparkle/PM6 $\mathrm{UME}_{a, b, c}=2.82 \%$ and $\mathrm{UME}_{\alpha, \beta, \gamma}=1.39 \%$ were obtained. Regarding the percent error for the volume, it was $25.5 \%$ for Sparkle/AM1, $3.60 \%$ for Sparkle/PM3, 6.82\% for Sparkle/PM6 and 2.01\% for Sparkle/PM7.

The size of the unit cell, having a minimum dimension larger than $8 \AA$, guarantees enough accuracy of the calculations according to the criteria given by Stewart [53]. Nevertheless, we repeated the calculations with $2 \times 1 \times 1,1 \times 2 \times 1,1 \times 1 \times 2,2 \times 2 \times 1$ and $2 \times 2 \times 2$ unit cells. In all cases, the results were consistent with those for the $1 \times 1 \times 1$ case (see supplementary information, Tables S1 to S6) with very similar values of $\mathrm{UME}_{a, b, c}$ and $\mathrm{UME}_{\alpha, \beta, \gamma}$ for each model at all computation sizes. For the two largest computations with $2 \times 2 \times 1$ and $2 \times 2 \times 2$ unit cells, the results from the $1 \times 1 \times 1$ computations for each model were used as pre-optimized initial conditions, which permitted a significant speedup of the calculations. In these two largest cases, either with or without preoptimization, the PM7 calculations showed worse behavior and failed to converge within the allowed time.

Apart from these accuracy considerations, it should also be noted that there are significant differences in the single-processor computation times, which range from 2 hours 11 minutes for Sparkle/AM1 to only 16 minutes for Sparkle/PM7 (for a single unit cell). These differences in the running times increase with the number of unit cells (e.g. for $2 \times 1 \times 1$, Sparkle/AM1 requires 8 hours 33 minutes, Sparkle/PM3 needs 12 hours 14 minutes and Sparkle/PM6 takes 6 hours 14 minutes, while Sparkle/PM7 requires only 1 hour 38 minutes). Consequently, Sparkle/PM7 not only provides higher accuracy than the other semi-empirical methods, but proves to be much faster.

We present now a more detailed comparison between the Sparkle/PM7 and crystallographic structures (see Figure 3) because the Sparkle/PM7 structure has the smallest difference from the experimental one. 


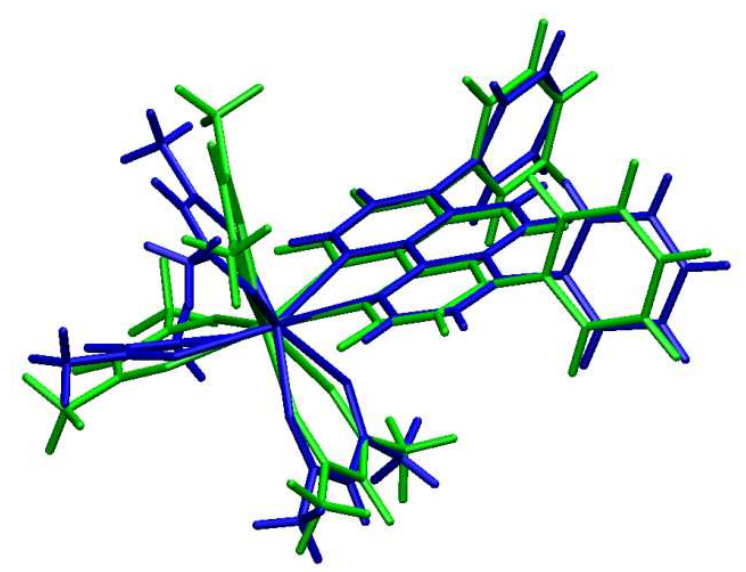

Figure 3. Comparison of the Sparkle/PM7 optimized geometry (green) with the X-ray geometry (blue) of the complex [Er(acac) $)_{3}$ (bath)] (software used for visualization: VMD version 1.9.1) [54].

The calculated Er-N distances, 2.471 and $2.473 \AA$, are slightly shorter than the experimental [2.545(2) and 2.550(2) $\AA$ ]. The same trend is observed in the calculated Er-O distances that lie in the 2.286-2.302 $\AA$ range. The $\mathrm{N}-\mathrm{Er}-\mathrm{N}$ bite angle is $66.0^{\circ}$, differing only $4 \%$ from the experimental value $\left[63.6^{\circ}\right]$. In the Sparkle/PM7 optimized geometry the terminal phenyl rings are rotated 73.68 and $74.20^{\circ}$ from the least squares plane of the phenanthroline unit. Overall, there is a good agreement between the experimental and the Sparkle/PM7 optimized geometry.

\subsection{X-ray powder diffraction}

A Rietveld refinement [55] was performed with Fullprof software [56] using the powderdiffraction data measured at room temperature. The overall parameters such as cell parameters, $2 \theta$ zero, scale factor, full-width at half-maximum and asymmetry parameter were allowed to refine in the range of 5 to $50^{\circ}$, with a final Bragg reliability factor of $14.4 \%$. The good agreement between experimental and calculated patterns (see Figure 4) confirms the high purity of the sample.

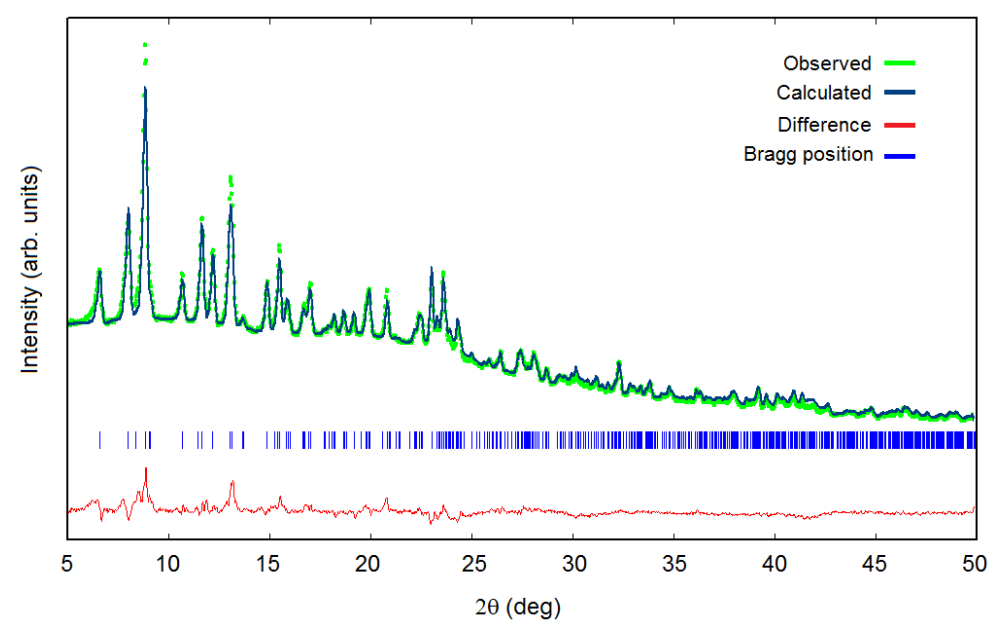

Figure 4. Results from a least-squares fit using the Rietveld method: experimental powder diffraction pattern (green); simulated diffraction pattern using the Rietveld method (dark-blue); difference between the observed and calculated intensities (red), and corresponding Bragg positions (blue). 


\subsection{Thermal analysis}

The TG, DTG and DSC curves for the studied complex, $\left[\operatorname{Er}(\mathrm{acac})_{3}(\mathrm{bath})\right]$, are depicted in Figure 5. The DSC shows an endothermic effect at $248{ }^{\circ} \mathrm{C}$, in agreement with the melting points reported for the analogous $\left[\mathrm{Tb}(\mathrm{acac})_{3}(\mathrm{phen})\right]$ and $\left[\mathrm{Eu}(\mathrm{acac})_{3}(\right.$ phen $\left.)\right]$ by Melby et al. [57]. As the temperature is further increased, partial decomposition (loss of bathophenanthroline ancillary ligand) takes place in two steps (evinced by two sharp DTG peaks at 283 and $369.6{ }^{\circ} \mathrm{C}$ ), with a weight loss of ca. $40 \%$ (calcd. weight loss for bath is $41 \%$ ). Above this temperature, gradual removal of acetylacetone units takes place. At $800^{\circ} \mathrm{C}$, the decomposition of the complex reaches ca. $50 \%$ weight loss, while that required for conversion into $\mathrm{Er}_{2} \mathrm{O}_{3}$ would be $78 \%$.

For comparison purposes, it is worth noting that aforementioned loss of the N,N-donor takes place at higher temperatures than those reported for analogous $\left[\operatorname{Pr}(\operatorname{acac})_{3}(\mathrm{phen})\right]$ and $\left[\mathrm{Nd}(\mathrm{acac})_{3}(\mathrm{phen})\right]$, in which the partial decomposition ends at $315-320^{\circ} \mathrm{C}[58,59]$. Thus, the bath complex is thermally more stable over its phen analogues, which in turn are more stable than their its bpy ones $[58,59]$. The reason for this could be related to the rigidly planar nature of bath.

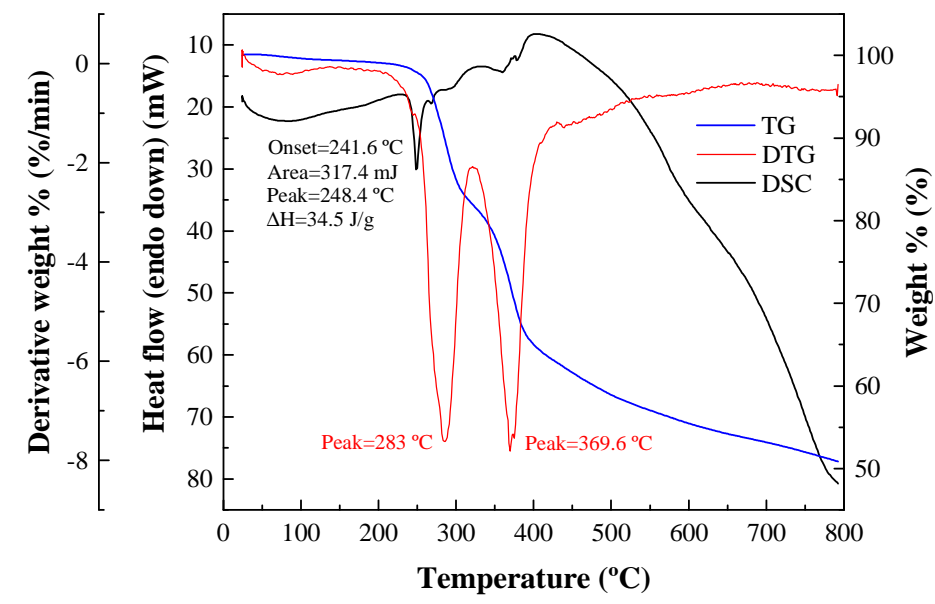

Figure 5. TG/DTG and DSC data for $\left[\operatorname{Er}(\mathrm{acac})_{3}(\mathrm{bath})\right]$ complex.

\subsection{Vibrational characterization}

\subsubsection{Infrared spectroscopy}

The FTIR spectrum (Figure 6) shows absorption bands with diverse intensities in the fingerprint region which correspond to main vibrations of bathophenanthroline and the acetylacetonate anions [60]. The carbonyl stretching frequency of the keto form of the ligand acac should appear at $1728 \mathrm{~cm}^{-1}$, while that of the enol form should appear at $1625 \mathrm{~cm}^{-1}$. These bands have been shifted to lower wavenumbers in the complex $\left(1620\right.$ and $\left.1599 \mathrm{~cm}^{-1}\right)$ indicating the involvement of carbonyl oxygen in the complex formation with the $\mathrm{Er}^{3+}$ ion. Furthermore, the red shift observed in the $\mathrm{C}=\mathrm{N}$ stretching frequencies of nitrogen donors (at $1519 \mathrm{~cm}^{-1}$ in the complex) shows the involvement of heteroaromatic nitrogen in the complex formation with the $\mathrm{Er}^{3+}$ ion [61].

Of particular interest is the series of absorption bands that are characteristic of the coordinated bathophenanthroline ancillary ligand and which appear at 764, 743, 703, 629 and $575 \mathrm{~cm}^{-1}$. It is also noteworthy that the band shown at $834 \mathrm{~cm}^{-1}$ (an aromatic $\mathrm{CH}$ out-of-plane bending vibration) is appreciably red shifted ( $v s$. that of free ligand) due to the perturbation induced by the coordination of this ligand to the $\mathrm{Er}^{3+}$ ion [62]. The band that appears at around $575 \mathrm{~cm}^{-1}$, which should assigned to a $v($ Er-N $)$ vibration, also offers evidence of the fact that the coordination bonds have been formed between erbium and bathophenathroline [63]. 
Finally, the low and broad absorption in the region 3500-2900 $\mathrm{cm}^{-1}$ corresponds to $\mathrm{O}-\mathrm{H}$ vibrations (due to atmospheric water absorption by $\mathrm{KBr}$ ) and $\mathrm{C}-\mathrm{H}$ vibrations.

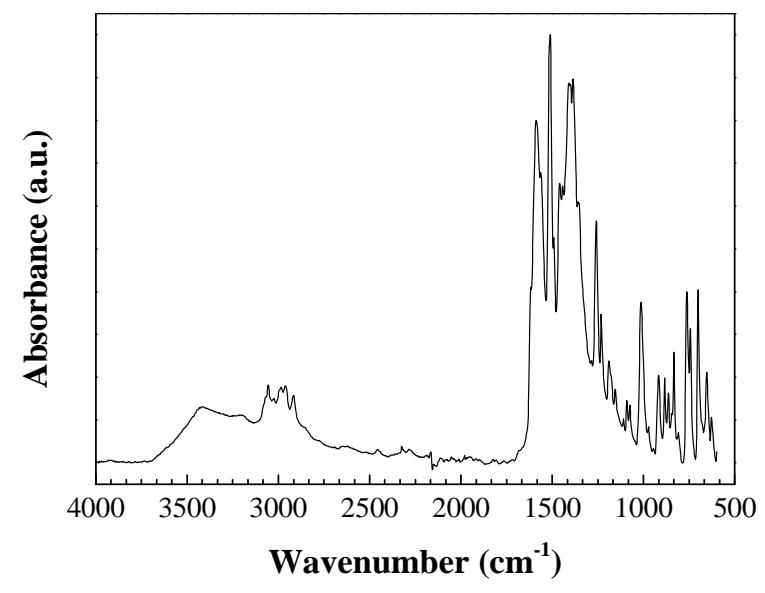

Figure 6. FTIR spectrum of $\left[\operatorname{Er}(\mathrm{acac})_{3}(\mathrm{bath})\right]$

\subsubsection{Raman spectroscopy}

The Raman spectrum of the complex (Figure 7) shows a strong multiple peak at $1600 \mathrm{~cm}^{-1}$, attributed to the $\mathrm{C}=\mathrm{N}$ and $\mathrm{C}=\mathrm{O}$ bonds from coordinated bathophenanthroline and the acetylacetonate anion. Peaks at $1446 \mathrm{~cm}^{-1}[v(\mathrm{C}=\mathrm{C}, \mathrm{C}=\mathrm{N})]$, at $1396 \mathrm{~cm}^{-1}[v(\mathrm{C}=\mathrm{C})+\delta(\mathrm{CH})]$ and near $700 \mathrm{~cm}^{-1}[\delta(\mathrm{CH})]$ are also characteristic of coordinated bathophenanthroline. Unassigned characteristic peaks of coordinated bathophenanthroline appear at 1290 and at $1000 \mathrm{~cm}^{-1}$.

The Raman-active peaks at approximately $408 \mathrm{~cm}^{-1}$ and that at $258 \mathrm{~cm}^{-1}$ can be tentatively assigned to $v(\mathrm{Er}-\mathrm{N})$ modes.

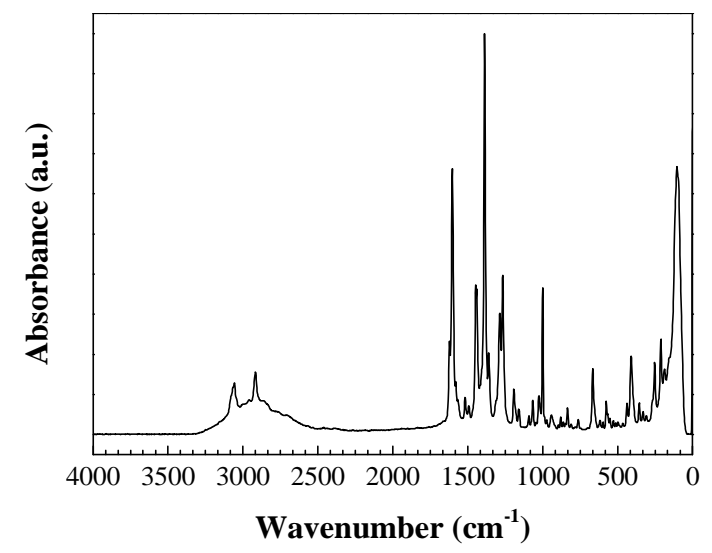

Figure 7. Raman spectrum of $\left[\operatorname{Er}(\mathrm{acac})_{3}(\right.$ bath $\left.)\right]$

\subsection{Absorption spectra}

\subsubsection{Experimental absorption spectra}

Figure 8 (left) shows the absorption spectrum in a $10^{-5} \mathrm{M}$ methanol diluted solution. The absorption spectrum is dominated by spin allowed $\pi-\pi^{*}$ transitions of the $\beta$-diketone and bathophenanthroline in the ultraviolet-visible region, with a maximum at $\lambda=280 \mathrm{~nm}[61,64]$. 
Absorption bands of Hacac and bath are red shifted in the complex and show stabilization of the ligand orbitals after complex formation [65]. Nonetheless, the spectral shapes of the complex and free ligands in solution are pretty similar, suggesting that the coordination of the $\mathrm{Er}^{3+}$ ion does not have a significant influence on the $\pi-\pi^{*}$ state energy [65].

Increasing the solution concentration up to $10^{-3} \mathrm{M}$ allows us to observe that the complex also exhibits the typical parity-forbidden narrow absorption bands, which originate from ground state ${ }^{4} \mathrm{I}_{15 / 2}$ to the various excited states of $\mathrm{Er}^{3+}$ ion, overlapped with the organic ligands absorption (Figure 8, inset). The transitions observed are (a) ${ }^{4} \mathrm{I}_{15 / 2} \rightarrow{ }^{2} \mathrm{G}_{11 / 2}(376 \mathrm{~nm}),\left(\right.$ b) ${ }^{4} \mathrm{I}_{15 / 2} \rightarrow\left({ }^{2} \mathrm{G},{ }^{4} \mathrm{~F},{ }^{2} \mathrm{H}\right)_{9 / 2}(405 \mathrm{~nm})$, (c) ${ }^{4} \mathrm{I}_{15 / 2} \rightarrow\left({ }^{4} \mathrm{~F}_{5 / 2},{ }^{4} \mathrm{~F}_{3 / 2}\right)(450 \mathrm{~nm}),\left(\right.$ d) ${ }^{4} \mathrm{I}_{15 / 2} \rightarrow{ }^{4} \mathrm{~F}_{7 / 2}$ (486.5 nm), (e) ${ }^{4} \mathrm{I}_{15 / 2} \rightarrow{ }^{2} \mathrm{H}_{11 / 2}$ (hypersensitive transition, $522 \mathrm{~nm})$, (f) ${ }^{4} \mathrm{I}_{15 / 2} \rightarrow{ }^{4} \mathrm{~S}_{3 / 2}(540 \mathrm{~nm}),(\mathrm{g}){ }^{4} \mathrm{I}_{15 / 2} \rightarrow{ }^{4} \mathrm{~F}_{9 / 2}(650.5 \mathrm{~nm})$ and (h) ${ }^{4} \mathrm{I}_{15 / 2} \rightarrow{ }^{4} \mathrm{I}_{11 / 2}(974.5$ nm, Figure 8, right) [66].
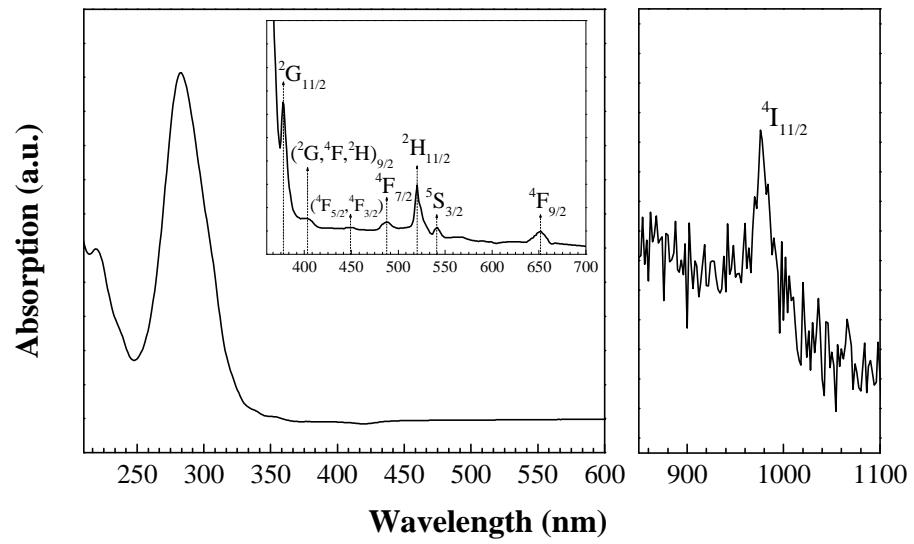

Figure 8. UV-Vis absorption spectra in $10^{-5} \mathrm{M}$ methanol solution (left) and in $10^{-3} \mathrm{M}$ methanol solution (inset, left); NIR absorption spectrum in $\mathrm{KBr}$ pellets (right).

\subsubsection{Calculated absorption spectra}

With a view to their practical applications, the ground state geometry of the molecule can be regarded irrelevant, while an accurate prediction of where the ligand-associated absorption occurs for these highly coordinated lanthanide complexes is crucial. Most popular ligands are UVexcitable [67], which can be unwanted and even harmful for living tissue, in the case of bio-sensing applications. Furthermore, no cheap pump sources are available in the UV [68]. Thus, one of the growing challenges in the chemistry of the lanthanide ions is to develop NIR luminescent lanthanide complexes that can be sensitized in the visible range $(>400 \mathrm{~nm})[69,70]$. Consequently, the ability to predict at which wavelength can a complex be excited is deemed crucial, and computational chemistry tools that can reliably model and predict the absorption and luminescent properties hold great potential so as to speed-up this materials design process.

As far as we know, there are no TDDFT studies of the absorption spectra of coordination compounds with erbium. For this reason, we attempted a real-time TDDFT calculation of the optical absorption spectrum of $\left[\operatorname{Er}(\mathrm{acac})_{3}(\right.$ bath $\left.)\right]$ in vacuum.

Spectra comparison. Regarding the use of semi-empirical calculations, the predicted spectra obtained with the INDO/S method and the different optimized geometries are very similar to each other and resemble the experimental one, except for the extra peak at $c a .250 \mathrm{~nm}$ (Figure 9). The approximations are acceptable for practical purposes for all Hamiltonians, albeit they display a systematic blue shift, which has been reported to range between 10 and $30 \mathrm{~nm}$ [71]. Conversely, the maximum of the TDDFT spectrum is strongly red-shifted in comparison with the experimental. This can be due to the limitations of TDDFT calculations for charge-transfer systems, such as the 
one under study. Although this is most likely the main reason behind the strong red-shift of the computed spectrum, the influence of the crystallographic environment cannot be neglected either. The calculations were done in the gas phase, albeit using the crystallographic geometry. Although technically not difficult, the optimization of the geometry of the full complex was not done, as it would lack physical meaning, since the absorption would then be computed for the gas phase geometry, while the experimental results were obtained for the bulk material. Not optimizing the geometry amounts to assuming that the main effect of the crystallographic environment of the complex on the absorption spectrum is the distortion of the complex geometry relative to the gas phase geometry. The impact of this possibly minute distortion can be assessed by comparing the spectra for the acetylacetonate ligand computed using the XRD geometry and for the optimized (gas phase) geometry (see Figure 10). This validates the assumption that the use of the unrelaxed geometry somehow mimics the main impact of the crystallographic environment. But the neglect of other effects, such as, e.g., environmental polarization, will certainly also contribute to the discrepancy between theoretical and experimental spectra.

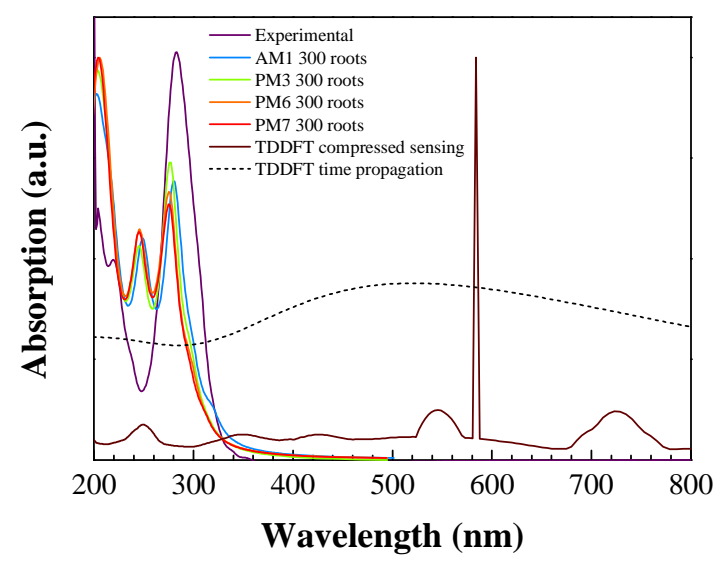

Figure 9. Comparison between the experimental (black line) and computed spectra of [ $\left.\operatorname{Er}(\mathrm{acac})_{3}(\mathrm{bath})\right]$, using semi-empirical methods (AM1, PM3, PM6 and PM7) and real-time TDDFT. The TDDFT spectrum was computed using compressed sensing techniques [49].

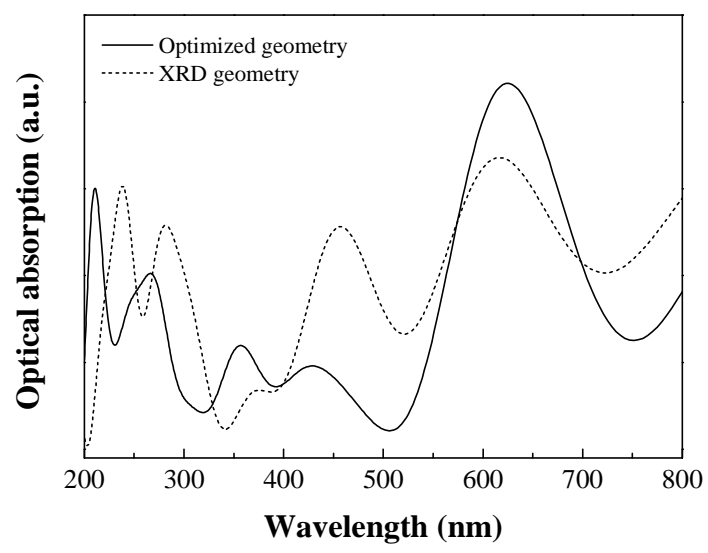

Figure 10. Absorption spectra for the acetylacetonate ligand using the XRD and the optimized (gas phase) geometries to assess the effect of the geometry optimization. 


\subsection{Excitation spectrum}

The excitation spectrum (Figure 11) consists of a broad band (from 325 to $450 \mathrm{~nm}$, with a maximum at ca. $378 \mathrm{~nm}$ ), associated to the excitation of the organic chromophores, and weaker intraconfigurational $f-f$ transitions, from the ${ }^{4} \mathrm{I}_{15 / 2}$ ground state to the ${ }^{4} \mathrm{~F}_{7 / 2}(486 \mathrm{~nm})$ and ${ }^{2} \mathrm{H}_{11 / 2}(522$ $\mathrm{nm})$ levels of the $\mathrm{Er}^{3+}$ ion, in good agreement with the spectra reported by Ilmi et al. for analogous $\mathrm{Sm}$ (III) and $\mathrm{Eu}$ (III) complexes [65]. The excitation spectrum reveals that the energy transfer from the ligands to $\mathrm{Er}^{3+}$ center is efficient, since the ligand-mediated excitation band is dominant in comparison to the $f-f$ transitions.

Nonetheless, it is worth noting that there is a significant shift of ca. $100 \mathrm{~nm}$ between the maximum of UV-Vis absorption spectrum and that of the excitation spectrum. According to Ilmi et al. [65], aforementioned broad band in the excitation spectrum would correspond to the triplet state of the ligand. The presence of such a $\pi-\pi^{*}$ spin-forbidden transition has been documented in the literature for acetylacetone [72]. According to Woo et al. [73], although absorption selection rules for photons forbid singlet-to-triplet transitions, it is known that, in the solid state, the excited states are not pure singlet or triplet states (and the excitation spectrum presented herein was collected in powder form). Furthermore, there is consensus on the presence of a triplet at that energy: Walzl et al. [74] reported that Hacac has a spin-forbidden transition beginning at $3.15 \mathrm{eV}$ with a maximum at $3.57 \mathrm{eV}$, that can either be attributed to $\pi-\pi^{*}$ singlet-triplet $(S-T)$ excitation in the acetylacetone enol or possibly to a $\pi-\pi^{*}(S-T)$ excitation in the acetylacetone keto form, very close to the $3.60 \mathrm{eV}$ value reported by Nakanishi et al. [75] for the acetylacetonate anion. Moreover, using the complete active space self-consistent field (CASSCF) and density functional theory (DFT) methods (CAS $(10,8)$ calculations), Chen et al. [76] determined the adiabatic excitation energy from $E-S_{0}$ to $E-T_{2}\left({ }^{3} n-\pi^{*}\right)$ to be $3.34 \mathrm{eV}$, very close to the $3.28 \mathrm{eV}$ value obtained from the experimental data. For comparison purposes, the positions (not the intensities) of the triplets have also been calculated by semiempirical methods (dashed lines in Figure 11), which display the systematic hypsochromic shift ( $v s$. the experimental spectrum) found in this type of calculations [71].

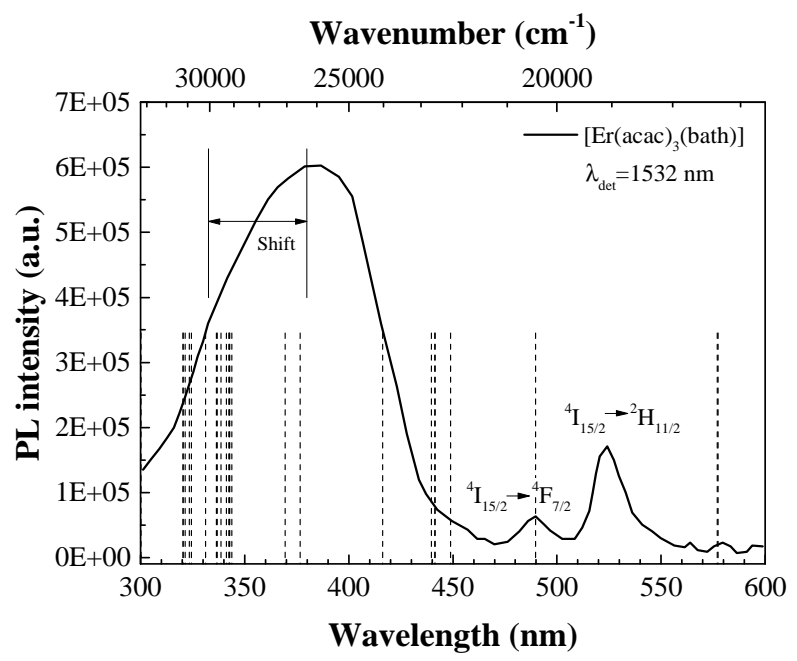

Figure 11. Excitation spectrum (solid line) and wavenumbers of the calculated triplet states (dashed lines, with arbitrary intensities). 


\subsection{Photoluminescence in solid state}

Because the molar absorption coefficients $\varepsilon$ of most of the transitions in the absorption spectra of the trivalent lanthanide ions are smaller than $10 \mathrm{~L} \cdot \mathrm{mol}^{-1} \cdot \mathrm{cm}^{-1}$, only a very limited amount of radiation is absorbed by direct excitation in the $4 f$ levels. Since the luminescence intensity is not only proportional to the luminescence quantum yield but also to the amount of light absorbed, weak light absorption results in weak luminescence. However, the problem of weak light absorption can be overcome by the so-called antenna effect (or sensitization) in which energy is transferred from the surroundings of the metal ion (i.e. from organic ligand via resonant energy transfer). In order to evidence the importance of this ligand sensitization effect, the emission spectra upon direct excitation of the $\mathrm{Er}^{3+}$ hypersensitive transition nearly $532 \mathrm{~nm}$ was also collected. The corresponding emission spectra (Figure 12) have been corrected for the different source intensities at $350 \mathrm{~nm}$ and at $532 \mathrm{~nm}$. It can be observed that the shape of the spectrum is the same in both cases, but upon excitation at $532 \mathrm{~nm}$ the PL intensity is half or a third than that gained upon excitation at $350 \mathrm{~nm}$.

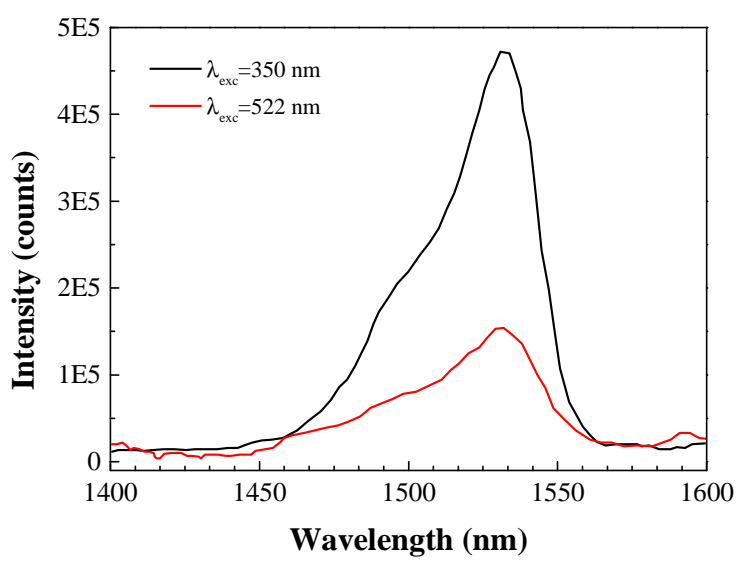

Figure 12. Comparison between emission spectra with excitation at $350 \mathrm{~nm}$ and at $522 \mathrm{~nm}$.

\subsection{Lifetime measurements}

The PL decay of the ${ }^{4} \mathrm{I}_{13 / 2}$ multiplet was measured after ${ }^{4} \mathrm{I}_{11 / 2}$ excitation (Figure 13). In spite of the possible PL reabsorption, the obtained decay shows single exponential behavior, with a time constant of $1.02 \mu \mathrm{s}$. This value, typical of lanthanide complexes [13,70,77] (and far smaller than the emission decay time of the isolated ion, $\tau \approx 8 \mathrm{~ms}$ ), is due to vibronic coupling with high energy $\mathrm{C}-\mathrm{H}$ stretching vibrations in the neighbourhood of the $\mathrm{Er}^{3+}$ ion (originated from the ligands), which lead to quenching of the excited state (because of the relatively small energy gap between the excited state ${ }^{4} \mathrm{I}_{13 / 2}$ and the ground state). For comparison purposes, this lifetime value is five times larger than that reported for erbium(III) tris (8-hydroxyquinolate) or $\operatorname{ErQ}_{3}(0.2 \mu \mathrm{s}$ in powder form) [47],

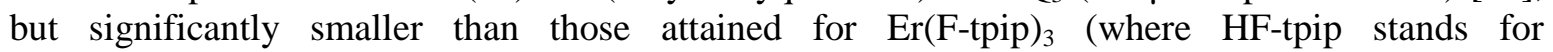
tetrapentafluorophenylimidodiphosphinate) [78] or for perfluorinated nitrosopyrazolone-based erbium chelates [79]: $164 \mu \mathrm{s}$ and $15.7 \mu \mathrm{s}$, respectively. 


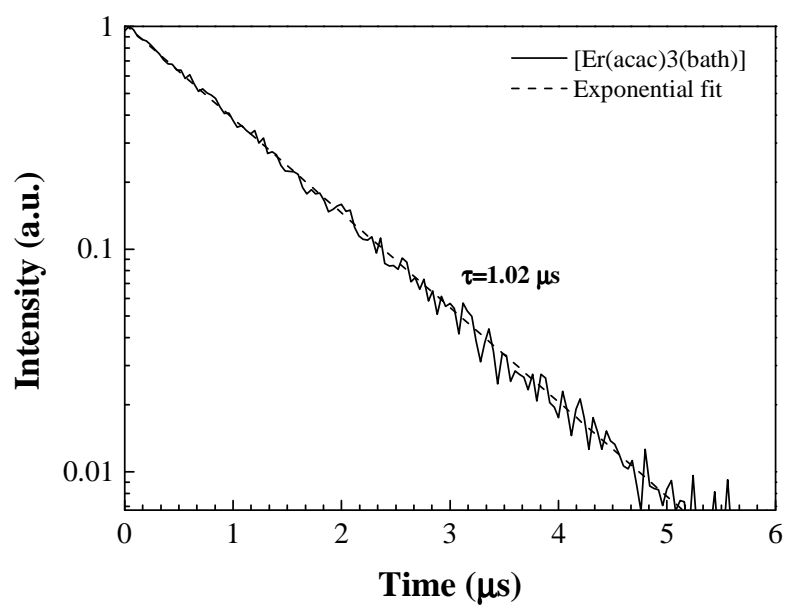

Figure 13. $1540 \mathrm{~nm}$ emission time decay measurement

\section{CONCLUSIONS}

Taking the simplest $\beta$-diketone, acetylacetone (Hacac), and a bulky $\mathrm{N}, \mathrm{N}$-donor (bathophenanthroline) as coordinating ligands, we have synthesized and thoroughly characterized a novel octacoordinated $\operatorname{Er}(\mathrm{III})$ complex, $\left[\mathrm{Er}(\mathrm{acac})_{3}(\mathrm{bath})\right]$. Subsequently, this data has been used as a reference in order to assess the goodness of several semi-empirical calculation methods for the modelling of its structure and spectroscopic properties. Further, a comparison with TDDFT results for the optical absorption spectrum has also been conducted.

From the experimental results we can conclude that the material shows efficient sensitization by antenna effect, leading to NIR emission at $1.53 \mu$ s from $\mathrm{Er}^{3+}$ ions upon ligand excitation in the UV, and good thermal stability.

With regard to the Sparkle/AM1, -PM3, -PM6 and -PM6 Hamiltonians evaluation, it can be inferred that PM7 Sparkle/PM7 not only provides higher accuracy than the other semi-empirical methods for the prediction of the ground state geometry, but also proves to be much faster. In relation the spectroscopic optical absorption spectrum estimation, the differences in the predicted spectra using the geometries optimized with the various Hamiltonians and the INDO/S method are small and can be regarded as an acceptable approximation for practical purposes in all cases. Consequently, Sparkle/PM7 can be deemed as a very promising tool for the design of novel Ln(III) complexes.

Conversely, the much more computationally-intensive TDDFT calculations have failed to deliver a reasonable approximation to the UV-Vis spectrum, most likely due to the limitations of these calculation for charge-transfer systems, such as the one under study.

\section{ACKNOWLEDGMENT}

The authors would like to thank Dr. M. Salvalaggio (Instituto Eni Donegani) and Prof. Carlos Zaldo (ICMM) for their help with the excitation spectrum and the lifetime measurements, respectively. P. Martín-Ramos and P. S. Pereira da Silva acknowledge Iberdrola Foundation and FCT (scholarship SFRH/BPD/84173/2012), respectively, for their financial support. CEMDRX group is grateful to Fundação para a Ciência e a Tecnologia (FCT) for providing funds under grant PEst-C/FIS/UI0036/2011. UVa group acknowledges financial support of Junta de Castilla y León through project VA300A12-1. 


\section{REFERENCES}

[1] A.Y. Rogachev, S.V. Konyukhov, A.V. Bochenkova, N.P. Kuzmina, A.V. Nemukhin, The QM/MM design of precursors for CVD and ALD processes, in: A. Devi (Ed.) EUROCVD 15: Fifteenth European Conference on Chemical Vapor Deposition : Proceedings of the International Symposium, Electrochemical Society, New Jersey, USA, 2005.

[2] J.D.L. Dutra, I.F. Gimenez, N.B.d.C. Junior, R.O. Freire, Theoretical design of highly luminescent europium (III) complexes: A factorial study, J. Photochem. Photobiol. A: Chem., 217 (2011) 389394.

[3] A.V. Nemukhin, A.Y. Rogachev, S.V. Konyukhov, A.V. Bochenkova, A.A. Granovsky, QM/MM modeling of the structures and properties of the $\beta$-diketonate-based lanthanide complexes, Int. J. Quantum Chem, 104 (2005) 203-213.

[4] P. Martín-Ramos, M.R. Silva, C. Coya, C. Zaldo, Á.L. Álvarez, S. Álvarez-García, A.M. Matos Beja, J. Martín-Gil, Novel erbium(III) fluorinated $\beta$-diketonate complexes with N,N-donors for optoelectronics: from synthesis to solution-processed devices, J. Mater. Chem. C, 1 (2013) 27252734.

[5] P. Martín-Ramos, V. Lavín, M. Ramos Silva, I.R. Martín, F. Lahoz, P. Chamorro-Posada, J.A. Paixão, J. Martín-Gil, Novel erbium(III) complexes with 2,6-dimethyl-3,5-heptanedione and different N,N-donor ligands for ormosil and PMMA matrices doping, J. Mater. Chem. C, 1 (2013) 5701-5710.

[6] P. Martín-Ramos, C. Coya, Á.L. Álvarez, M. Ramos Silva, C. Zaldo, J.A. Paixão, P. ChamorroPosada, J. Martín-Gil, Charge transport and sensitized $1.5 \mu \mathrm{m}$ electroluminescence properties of full solution-processed NIR-OLED based on novel $\operatorname{Er}(\mathrm{III})$ fluorinated $\beta$-diketonate ternary complex, J. Phys. Chem. C, 117 (2013) 10020-10030.

[7] P. Martín-Ramos, P.S. Pereira da Silva, V. Lavín, I.R. Martín, F. Lahoz, P. Chamorro-Posada, M. Ramos Silva, J. Martín-Gil, Structure and NIR-luminescence of ytterbium(III) beta-diketonate complexes with 5-nitro-1,10-phenanthroline ancillary ligand: assessment of chain length and fluorination impact, Dalton Trans., 42 (2013) 13516-13526.

[8] M. Ramos Silva, P. Martín-Ramos, J.T. Coutinho, L.C.J. Pereira, J. Martín-Gil, Effect of the capping ligand on luminescent erbium(III) $\beta$-diketonate single-ion magnets, Dalton Trans., 43 (2014) 6752-6761.

[9] P. Martín-Ramos, M.C. Coya-Párraga, V. Lavín, I.R. Martín, M. Ramos Silva, P.S. Pereira Silva, M. García-Vélez, Á.L. Álvarez, J. Martín-Gil, Active-layer solution-processed NIR-OLEDs based on ternary erbium(III) complexes with 1,1,1-trifluoro-2,4-pentanedione and different N,N-donors, Dalton Trans., 43 (2014) 18087-18096.

[10] M. Ramos Silva, P. Martín-Ramos, J.T. Coutinho, L.C.J. Pereira, V. Lavín, I.R. Martín, P.S. Pereira Silva, J. Martín-Gil, Slow magnetic relaxation mechanisms in Erbium SIMs, Dalton Trans., 44 (2015) 1264-1272.

[11] P. Martín-Ramos, M. Ramos Silva, J.T. Coutinho, L.C.J. Pereira, P. Chamorro-Posada, J. MartínGil, Single-Ion Magnetism in a Luminescent $\mathrm{Er}^{3+} \beta$-Diketonato Complex with Multiple Relaxation Mechanisms, Eur. J. Inorg. Chem., 2014 (2014) 511-517.

[12] S. Comby, J.-C.G. Bünzli, Lanthanide Near-Infrared Luminescence in Molecular Probes and Devices, in: V.K. Pecharsky, J.C.G. Bünzli, K.A. Gschneider, Jr. (Eds.) Optical Spectroscopy, Elsevier, 2007, pp. 217-470.

[13] Z. Li, J. Yu, L. Zhou, H. Zhang, R. Deng, Z. Guo, $1.54 \mu \mathrm{m}$ Near-infrared photoluminescent and electroluminescent properties of a new Erbium (III) organic complex, Org. Electron., 9 (2008) 487494. 
[14] R. Van Deun, D. Moors, B. De Fré, K. Binnemans, Near-infrared photoluminescence of lanthanidedoped liquid crystals, J. Mater. Chem., 13 (2003) 1520.

[15] L.N. Sun, H.J. Zhang, L.S. Fu, F.Y. Liu, Q.G. Meng, C.Y. Peng, J.B. Yu, A New Sol-Gel Material Doped with an Erbium Complex and Its Potential Optical-Amplification Application, Adv. Funct. Mater., 15 (2005) 1041-1048.

[16] P. Lenaerts, K. Driesen, R. Van Deun, K. Binnemans, Covalent Coupling of Luminescent Tris(2thenoyltrifluoroacetonato)lanthanide(III) Complexes on a Merrifield Resin, Chem. Mater., 17 (2005) 2148-2154.

[17] P. Lenaerts, E. Ryckebosch, K. Driesen, R. Van Deun, P. Nockemann, C. Görller-Walrand, K. Binnemans, Study of the luminescence of tris(2-thenoyltrifluoroacetonato)lanthanide(III) complexes covalently linked to 1,10-phenanthroline-functionalized hybrid sol-gel glasses, J. Lumin., 114 (2005) 77-84.

[18] P. Lenaerts, A. Storms, J. Mullens, J. D'Haen, C. Görller-Walrand, K. Binnemans, K. Driesen, Thin Films of Highly Luminescent Lanthanide Complexes Covalently Linked to an Organic-Inorganic Hybrid Material via 2-Substituted Imidazo[4,5-f]-1,10-phenanthroline Groups, Chem. Mater., 17 (2005) 5194-5201.

[19] S. Moynihan, R. Van Deun, K. Binnemans, G. Redmond, Optical properties of planar polymer waveguides doped with organo-lanthanide complexes, Opt. Mater., 29 (2007) 1821-1830.

[20] S. Moynihan, R. Van Deun, K. Binnemans, J. Krueger, G. von Papen, A. Kewell, G. Crean, G. Redmond, Organo-lanthanide complexes as luminescent dopants in polymer waveguides fabricated by hot embossing, Opt. Mater., 29 (2007) 1798-1808.

[21] M. Irfanullah, K. Iftikhar, Hypersensitivity in the Luminescence and 4f-4f Absorption Properties of Mono- and Dinuclear Eu ${ }^{\mathrm{III}}$ and $\mathrm{Er}^{\mathrm{III}}$ Complexes Based on Fluorinated $\beta$-Diketone and Diimine/BisDiimine Ligands, J. Fluoresc., 21 (2010) 81-93.

[22] X.-D. Yang, W.-B. Chang, Y.-X. Ci, Time-resolved fluorescence immunoassay with measurement of a europium chelate in solution: dissociation conditions and application for determination of cortisol, Anal. Chem., 66 (1994) 2590-2594.

[23] Y.S. Yang, M.L. Gong, Y.Y. Li, H.Y. Lei, S.L. Wu, Effects of the structure of ligands and their $\mathrm{Ln}^{3+}$ complexes on the luminescence of the central $\mathrm{Ln}^{3+}$ ions, J. Alloys Compd., 207-208 (1994) $112-114$.

[24] M. Irfanullah, K. Iftikhar, Photoluminescence, optical absorption and hypersensitivity in mono- and dinuclear lanthanide $\left(\mathrm{Tb}^{\mathrm{III}}\right.$ and $\mathrm{Ho}^{\mathrm{III}}$ ) $\beta$-diketonate complexes with diimines and bis-diimine bridging ligand, J. Lumin., 130 (2010) 1983-1993.

[25] G. Sheldrick, SADABS, in, University of Göttingen, Göttingen, Germany, 1996.

[26] G.M. Sheldrick, A short history of SHELX, Acta Crystallogr. Sect. A: Found. Crystallogr., 64 (2007) 112-122.

[27] A.L. Spek, Single-crystal structure validation with the program PLATON, J. Appl. Crystallogr., 36 (2003) 7-13.

[28] C.F. Macrae, P.R. Edgington, P. McCabe, E. Pidcock, G.P. Shields, R. Taylor, M. Towler, J. van de Streek, Mercury: visualization and analysis of crystal structures, J. Appl. Crystallogr., 39 (2006) 453-457.

[29] M.J.S. Dewar, E.G. Zoebisch, E.F. Healy, J.J.P. Stewart, Development and use of quantum mechanical molecular models. 76. AM1: a new general purpose quantum mechanical molecular model, J. Am. Chem. Soc., 107 (1985) 3902-3909.

[30] R.O. Freire, E.V. do Monte, G.B. Rocha, A.M. Simas, AM1 Sparkle modeling of Er(III) and Ce(III) coordination compounds, J. Organomet. Chem., 691 (2006) 2584-2588.

[31] J.J.P. Stewart, Optimization of parameters for semiempirical methods I. Method, J. Comput. Chem., 10 (1989) 209-220. 
[32] J.J.P. Stewart, Optimization of parameters for semiempirical methods V: Modification of NDDO approximations and application to 70 elements, J. Mol. Model., 13 (2007) 1173-1213.

[33] R.O. Freire, A.M. Simas, Sparkle/PM6 Parameters for all Lanthanide Trications from La(III) to Lu(III), J. Chem. Theory Comput., 6 (2010) 2019-2023.

[34] J.J.P. Stewart, Optimization of parameters for semiempirical methods VI: more modifications to the NDDO approximations and re-optimization of parameters, J. Mol. Model., 19 (2012) 1-32.

[35] J.D.L. Dutra, M.A.M. Filho, G.B. Rocha, R.O. Freire, A.M. Simas, J.J.P. Stewart, Sparkle/PM7 Lanthanide Parameters for the Modeling of Complexes and Materials, J. Chem. Theory Comput., 9 (2013) 3333-3341.

[36] J.J.P. Stewart, MOPAC2012, in, Stewart Computational Chemistry, Colorado Springs, CO, USA, 2012.

[37] F. Neese, The ORCA program system, Wiley Interdisciplinary Reviews: Computational Molecular Science, 2 (2012) 73-78.

[38] T. Petrenko, F. Neese, Analysis and prediction of absorption band shapes, fluorescence band shapes, resonance Raman intensities, and excitation profiles using the time-dependent theory of electronic spectroscopy, J. Chem. Phys., 127 (2007) 164319.

[39] J. Ridley, M. Zerner, An intermediate neglect of differential overlap technique for spectroscopy: Pyrrole and the azines, Theor. Chim. Acta, 32 (1973) 111-134.

[40] M.C. Zerner, G.H. Loew, R.F. Kirchner, U.T. Mueller-Westerhoff, An intermediate neglect of differential overlap technique for spectroscopy of transition-metal complexes. Ferrocene, J. Am. Chem. Soc., 102 (1980) 589-599.

[41] A.V.M. de Andrade, R.L. Longo, A.M. Simas, G.F. de Sá, Theoretical model for the prediction of electronic spectra of lanthanide complexes, J. Chem. Soc., Faraday Trans., 92 (1996) 1835.

[42] C.A.F. de Oliveira, F.F. da Silva, I. Malvestiti, V.R.d.S. Malta, J.D.L. Dutra, N.B. da Costa, R.O. Freire, S.A. Júnior, Effect of temperature on formation of two new lanthanide metal-organic frameworks: Synthesis, characterization and theoretical studies of Tm(III)-succinate, J. Solid State Chem., 197 (2013) 7-13.

[43] Fundamentals of time-dependent density functional theory, Springer Verlag, Berlin, 2012.

[44] A. Castro, H. Appel, M. Oliveira, C.A. Rozzi, X. Andrade, F. Lorenzen, M.A.L. Marques, E.K.U. Gross, A. Rubio, octopus: a tool for the application of time-dependent density functional theory, physica status solidi (b), 243 (2006) 2465-2488.

[45] X. Andrade, J. Alberdi-Rodriguez, D.A. Strubbe, M.J.T. Oliveira, F. Nogueira, A. Castro, J. Muguerza, A. Arruabarrena, S.G. Louie, A. Aspuru-Guzik, A. Rubio, M.A.L. Marques, Timedependent density-functional theory in massively parallel computer architectures: the octopus project, J. Phys.: Condens. Matter, 24 (2012) 233202.

[46] M.J.T. Oliveira, F. Nogueira, Generating relativistic pseudo-potentials with explicit incorporation of semi-core states using APE, the Atomic Pseudo-potentials Engine, Comput. Phys. Commun., 178 (2008) 524-534.

[47] N. Troullier, J.L. Martins, Efficient pseudopotentials for plane-wave calculations, Physical Review B, 43 (1991) 1993-2006.

[48] J.P. Perdew, Self-interaction correction to density-functional approximations for many-electron systems, Physical Review B, 23 (1981) 5048-5079.

[49] X. Andrade, J.N. Sanders, A. Aspuru-Guzik, Application of compressed sensing to the simulation of atomic systems, Proceedings of the National Academy of Sciences, 109 (2012) 13928-13933.

[50] S. Cheng, F. Yuguo, L. Guofa, W. Yutian, L. Pinzhe, Gaodeng Xuexiao Huaxue Xuebao (Chin.) Chem. J. Chinese U., (1983) 769.

[51] S.-D. Jiang, B.-W. Wang, G. Su, Z.-M. Wang, S. Gao, A Mononuclear Dysprosium Complex Featuring Single-Molecule-Magnet Behavior, Angew. Chem. Int. Ed., 49 (2010) 7448-7451. 
[52] G.M. Neelgund, S.A. Shivashankar, T. Narasimhamurthy, R.S. Rathore, Tris(acetylacetonato$\left.\kappa 2 \mathrm{O}, \mathrm{O}^{\prime}\right)\left(1,10\right.$-phenanthroline- $\left.\kappa 2 \mathrm{~N}, \mathrm{~N}^{\prime}\right)$ erbium(III), Acta Crystallographica Section C Crystal Structure Communications, 63 (2007) m74-m76.

[53] J.J.P. Stewart, Application of the PM6 method to modeling the solid state, J. Mol. Model., 14 (2008) 499-535.

[54] W. Humphrey, A. Dalke, K. Schulten, VMD: Visual molecular dynamics, J. Mol. Graphics, 14 (1996) 33-38.

[55] R.A. Young, The Rietveld Method, Oxford University Press, 1995.

[56] J. Rodríguez-Carvajal, Recent advances in magnetic structure determination by neutron powder diffraction, Physica B: Condensed Matter, 192 (1993) 55-69.

[57] L.R. Melby, N.J. Rose, E. Abramson, J.C. Caris, Synthesis and Fluorescence of Some Trivalent Lanthanide Complexes, J. Am. Chem. Soc., 86 (1964) 5117-5125.

[58] A.A. Ansari, M. Irfanullah, K. Iftikhar, Optical absorption and NMR spectroscopic studies on paramagnetic neodymium(III) complexes with $\beta$-diketone and heterocyclic amines, Spectrochimica Acta Part A: Molecular and Biomolecular Spectroscopy, 67 (2007) 1178-1188.

[59] A.A. Ansari, Z. Ahmed, K. Iftikhar, Nuclear magnetic resonance and optical absorption spectroscopic studies on paramagnetic praseodymium(III) complexes with $\beta$-diketone and heterocyclic amines, Spectrochimica Acta Part A: Molecular and Biomolecular Spectroscopy, 68 (2007) 176-183.

[60] F. Marandi, R. Rutvand, M. Rafiee, J.H. Goh, H.-K. Fun, Synthesis, properties and crystal structures of new binuclear lead(II) complexes based on phenyl, naphthyl-containing fluorine $\beta$ diketones and substituted 2,2'-bipyridines, Inorg. Chim. Acta, 363 (2010) 4000-4007.

[61] H. Gallardo, G. Conte, A.J. Bortoluzzi, I.H. Bechtold, A. Pereira, W.G. Quirino, C. Legnani, M. Cremona, Synthesis, structural characterization, and photo and electroluminescence of a novel terbium(III) complex: $\quad\{$ Tris(acetylacetonate) $\quad[1,2,5]$ thiadiazolo[3,4f][1,10]phenanthroline \}terbium(III), Inorg. Chim. Acta, 365 (2011) 152-158.

[62] G.B. Deacon, D.J. Evans, P.C. Junk, New Variations on the $\mathrm{LnCl}_{3}(\mathrm{~L})_{\mathrm{n}}(\mathrm{L}=$ tetrahydrofuran or 1,2dimethoxyethane) Structural Theme $-\mathrm{NdCl}_{3}(\mathrm{dme})_{2}$ and $\mathrm{YbCl}_{3}(\text { thf })_{3.5}$, Z. Anorg. Allg. Chem., 628 (2002) 2033-2036.

[63] L.-J. Bian, H.-A. Xi, X.-F. Qian, J. Yin, Z.-K. Zhu, Q.-H. Lu, Synthesis and luminescence property of rare earth complex nanoparticles dispersed within pores of modified mesoporous silica, Mater. Res. Bull., 37 (2002) 2293-2301.

[64] R.L. Lintvedt, L.K. Kernitsky, Ligand field information from charge-transfer spectra of substituted tris(1,3-diketonato)iron(III) chelates. Spectrochemical series for 1,3-diketones, Inorg. Chem., 9 (1970) 491-494.

[65] R. Ilmi, K. Iftikhar, Synthesis, visible light absorption and luminescence of bipyrimidine bridged dinuclear lanthanide complexes of 2,4-pentanedione, Inorg. Chem. Commun., 13 (2010) 15521557.

[66] C. Görller-Walrand, K. Binnemans, Spectral intensities of f-f transitions, in: K.A. Gschneidner, L. Eyring (Eds.) Handbook on the physics and chemistry of rare earths, Elsevier BV, Amsterdam, 1998, pp. 101-264.

[67] B. Yan, Recent progress in photofunctional lanthanide hybrid materials, RSC Adv., 2 (2012) 9304.

[68] K. Kuriki, Y. Koike, Y. Okamoto, Plastic Optical Fiber Lasers and Amplifiers Containing Lanthanide Complexes, Chem. Rev., 102 (2002) 2347-2356.

[69] V. Divya, R.O. Freire, M.L.P. Reddy, Tuning of the excitation wavelength from UV to visible region in $\mathrm{Eu}^{3+}-\beta$-diketonate complexes: Comparison of theoretical and experimental photophysical properties, Dalton Trans., 40 (2011) 3257. 
[70] P. Martín-Ramos, I.R. Martín, F. Lahoz, S. Hernández-Navarro, P.S. Pereira da Silva, I. Hernández-Campo, V. Lavín, M. Ramos Silva, An erbium(III)-based NIR emitter with a highly conjugated $\beta$-diketonate for blue-region sensitization, J. Alloys Compd., 619 (2015) 553-559.

[71] G.F. de Sá, O.L. Malta, C. de Mello Donegá, A.M. Simas, R.L. Longo, P.A. Santa-Cruz, E.F. da Silva, Spectroscopic properties and design of highly luminescent lanthanide coordination complexes, Coord. Chem. Rev., 196 (2000) 165-195.

[72] V. Mohaček-Grošev, K. Furić, H. Ivanković, Luminescence and Raman Spectra of Acetylacetone at Low Temperatures, The Journal of Physical Chemistry A, 111 (2007) 5820-5827.

[73] H. Woo, S. Graham, D. Halliday, D. Bradley, R. Friend, P. Burn, A. Holmes, Photoinduced absorption and photoluminescence in poly(2,5-dimethoxy-p-phenylene vinylene), Physical Review B, 46 (1992) 7379-7389.

[74] K.N. Walzl, I.M. Xavier, A. Kuppermann, Electron-impact spectroscopy of various diketone compounds, The Journal of Chemical Physics, 86 (1987) 6701.

[75] H. Nakanishi, H. Morita, S. Nagakura, Electronic Structures and Spectra of the Keto and Enol Forms of Acetylacetone, Bull. Chem. Soc. Jpn., 50 (1977) 2255-2261.

[76] X.-B. Chen, W.-H. Fang, D.L. Phillips, Theoretical Studies of the Photochemical Dynamics of Acetylacetone: Isomerzation, Dissociation, and Dehydration Reactions, The Journal of Physical Chemistry A, 110 (2006) 4434-4441.

[77] X. Li, Z. Si, C. Pan, L. Zhou, Z. Li, X. Li, J. Tang, H. Zhang, Near-infrared luminescent properties and natural lifetime calculation of a novel $\mathrm{Er}^{3+}$ complex, Inorg. Chem. Commun., 12 (2009) 675677.

[78] G. Mancino, A.J. Ferguson, A. Beeby, N.J. Long, T.S. Jones, Dramatic Increases in the Lifetime of the $\mathrm{Er}^{3+}$ Ion in a Molecular Complex Using a Perfluorinated Imidodiphosphinate Sensitizing Ligand, J. Am. Chem. Soc., 127 (2005) 524-525.

[79] L. Beverina, M. Crippa, M. Sassi, A. Monguzzi, F. Meinardi, R. Tubino, G.A. Pagani, Perfluorinated nitrosopyrazolone-based erbium chelates: a new efficient solution processable NIR emitter, Chem. Commun., (2009) 5103. 